\title{
Equivalence of Boltzmann and moment equations (Corrigendum)
}

\author{
Rafael Cubarsi
}

\begin{abstract}
Dept. de Matemàtiques, Universitat Politècnica de Catalunya, 08034 Barcelona, Spain e-mail: rafael. cubarsi@upc.edu
\end{abstract}

A\&A 522, A30 (2010), DOI: 10.1051/0004-6361/201014766

Key words. stars: kinematics and dynamics - Galaxy: kinematics and dynamics - galaxies: statistics - methods: analytical hydrodynamics - errata, addenda

In Cubarsi (2010)'s Eq. (15) relating the symmetric tensor components $\Lambda_{i_{1} \ldots i_{k}}$ and $\lambda_{i_{1} i_{2} \ldots i_{k}}$ was not correctly written. It should be

$$
\begin{aligned}
& \Lambda_{0}=\frac{\partial \lambda_{0}}{\partial t}-\frac{\partial \mathcal{U}}{\partial r_{i}} \lambda_{i} \\
& \Lambda_{i}=\frac{\partial \lambda_{i}}{\partial t}+\frac{\partial \lambda_{0}}{\partial r_{i}}-2 \frac{\partial \mathcal{U}}{\partial r_{k}} \lambda_{i k} \\
& \Lambda_{i j}=\frac{\partial \lambda_{i j}}{\partial t}+\mathcal{S}\left(\frac{\partial \lambda_{i}}{\partial r_{j}}\right)-3 \frac{\partial \mathcal{U}}{\partial r_{k}} \lambda_{i j k}, \\
& \ldots \\
& \Lambda_{i_{1} \ldots i_{n-1}}=\frac{\partial \lambda_{i_{1} i_{2} \ldots i_{n-1}}}{\partial t}+\mathcal{S}\left(\frac{\partial \lambda_{i_{1} i_{2} \ldots i_{n-2}}}{\partial r_{n-1}}\right)-n \frac{\partial \mathcal{U}}{\partial r_{i_{n}}} \lambda_{i_{1} i_{2} \ldots i_{n}}, \\
& \Lambda_{i_{1} \ldots i_{n}}=\frac{\partial \lambda_{i_{1} i_{2} \ldots i_{n}}}{\partial t}+\mathcal{S}\left(\frac{\partial \lambda_{i_{1} i_{2} \ldots i_{n-1}}}{\partial r_{i_{n}}}\right) \\
& \Lambda_{i_{1} \ldots i_{n+1}}=\mathcal{S}\left(\frac{\partial \lambda_{i_{1} i_{2} \ldots i_{n}}}{\partial r_{i_{n+1}}}\right)
\end{aligned}
$$

where $\mathcal{S}$ means the symmetrized tensor.

They are now symmetric quantities, all the results remain totally valid.

\section{References}

Cubarsi, R. 2010, A\&A, 522, A30 